Church of Rilán: evidence of prefabrication in the "Chilota School of Architecture" in wood

\section{| RESUMEN |}

Durante la última restauración integral de la Iglesia de Rilán llevada a cabo por la Fundación Amigos de las Iglesias de Chiloé (FAICH), encontramos un hallazgo histórico: todas las piezas estructurales de esta iglesia declarada Patrimonio de la Humanidad estaban talladas con números romanos. Este hecho nos plantea una serie de hipótesis que van desde la prefabricación como parte de la evolución del sistema constructivo a la posibilidad que esta Escuela Chilota de Carpintería haya alcanzado una avanzada técnica, existiendo grupos especializados de carpinteros. Este documento es una invitación a la investigación del hecho, ya que de comprobarse nuestras teorías, podría permitir conocer una nueva etapa en la construcción de templos chilotes, que hasta ahora solo se basaba en el concepto de minga.

\section{| ABSTRACT |}

During the last comprehensive restoration of Rilán's Church carried out by the Foundation, we made a historical finding: all the structural pieces of this church -declared Heritage of Humanitywere marked with roman numbers. This fact raises a series of hypotheses, which range from the prefabrication as part of the evolution of the constructive system, to the possibility that this Chiloé School of Carpentry reached an advanced technic that featured specialized groups of carpenters. This document is an invitation to conduct research on the topic, since by verifying our theories, it might allow discovering a new stage in the construction of Chiloé's temples that, so far has only been based on the concept of "minga".

\title{
Iglesia de Rilán: indicios de prefabricación en la Escuela Chilota de arquitectura en madera
}

En el año 1976, el Obispado de Ancud firmó E un convenio con la Facultad de Arquitectura y Urbanismo de la Universidad de Chile bajo el nombre de "Programa de protección y desarrollo del patrimonio arquitectónico de Chiloé", el cual dio origen a un gran número de actividades que permitieron tomar conciencia sobre la riqueza del patrimonio arquitectónico de este territorio, creando un ambiente favorable en su defensa. En 1993 como consecuencia de esta cooperación y ante la necesidad de obtener y administrar fondos para la conservación de dicho patrimonio, se crea la Fundación de Amigos de las Iglesias de Chiloé (FAICH), una organización sin fines de lucro dependiente del Obispado de Ancud, que tiene como objetivo conservar, preservar y difundir el patrimonio cultural de Chiloé.

Este patrimonio, tangible e intangible, se ve expresado nítidamente en la "Escuela Chilota de Arquitectura Religiosa en Madera", el cual dio forma a las cientos de iglesias chilotas -de las que hoy quedan cerca de 70-, levantadas mediante un sistema constructivo y una tipología particular, nacidas del conocimiento del medio y diestro manejo de la madera. Entre los años 2000 y 2001, la Unesco declaró Patrimonio de la Humanidad a 16 de estas Iglesias de Chiloé.
Durante todos estos años, la FAICH ha restaurado de manera parcial e integral 11 iglesias, siguiendo un riguroso procedimiento de alzaprimado en toda la estructura, siendo posible el desmonte y reemplazo de piezas que así lo requieran, sin desarmar completamente la iglesia. Este sistema y en general todos los procedimientos que utiliza la Fundación son trabajados en conjunto y aprobados por el Consejo de Monumentos Nacionales (CMN), basados en el documento "Criterios de Intervención de las Iglesias de Chiloé".

Sin embargo, en la restauración integral de la iglesia de Rilán (2012-2013), el avanzado y grave deterioro provocado por agentes xilófagos y hongos de pudrición presentes en la estructura y revestimientos no nos permitió llevar a cabo este procedimiento, debido al gran riesgo que implicaba el desarme en la seguridad de la iglesia y los trabajadores, por lo que se decidió, con la aprobación de CMN, desarmar la iglesia por etapas, desde la torre a la culata, retirando revestimientos interiores, exteriores y piezas estructurales, las cuales fueron igualmente almacenadas y clasificadas y diagnosticadas.

Durante el proceso de desarme de la torre, al bajar la solera superior de la segunda caña

\footnotetext{
* Arquitecto Universidad Austral, Jefe de obra de la Restauración Iglesia Santa María de Rilán.

** Arquitecto Universidad de Chile, Asistente de obra de la Restauración Iglesia Santa María de Rilán.
} 
1. Esquema ilustrativo de los cuerpos y su respectiva numeración, según las marcas encontradas en la estructura de la iglesia al momento del desarme.

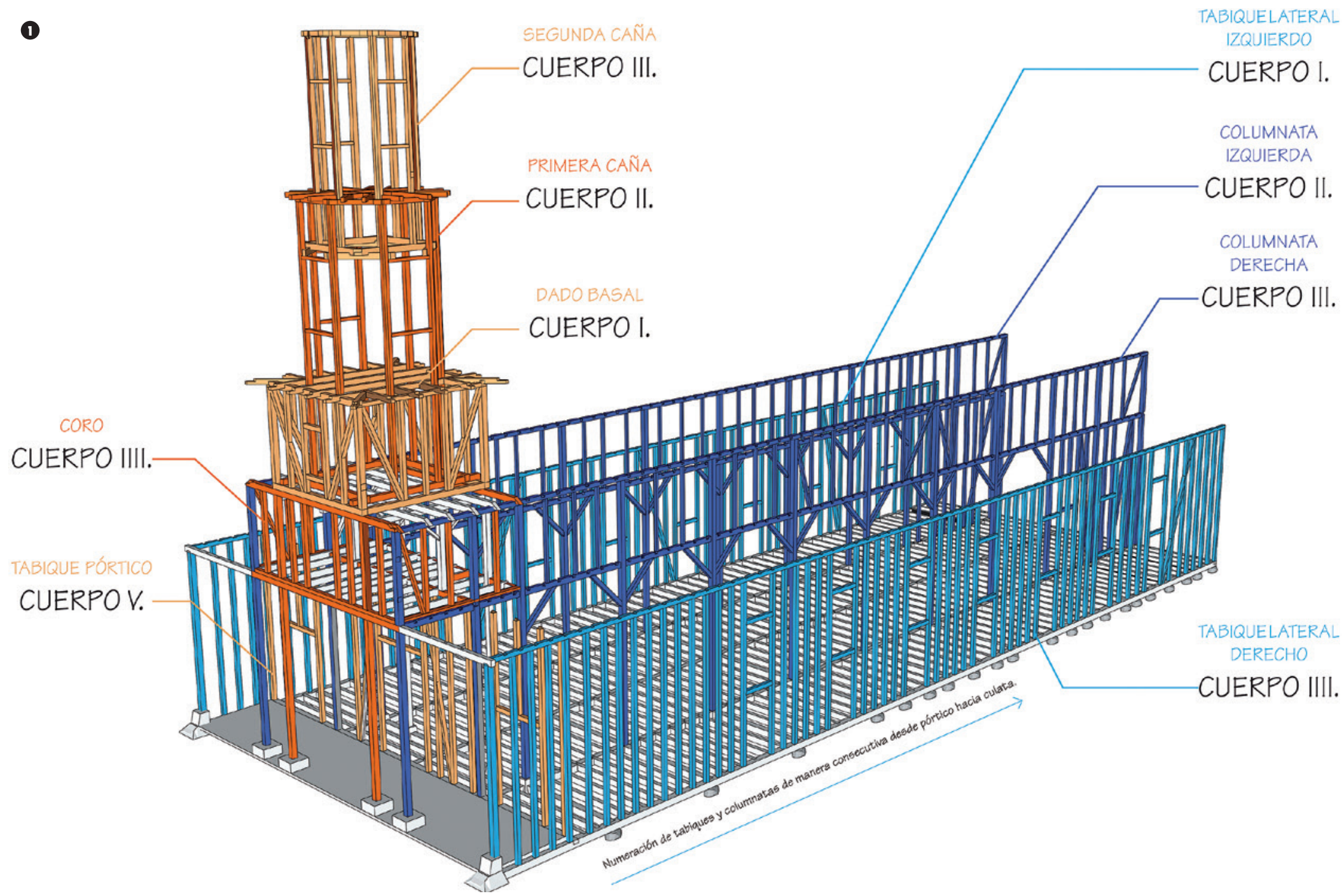

al nivel del suelo y rearmarla para constatar la existencia y daño general de todas sus piezas, nos percatamos de algo fuera de lo común en otros desarmes. Las ocho piezas que conformaban dicha solera presentaban un tallado que evidenciaba un código con dos números romanos en cada sección: un número común para todas y otro correlativo que indicaba la posición de las piezas. Quedamos atentos a la posible aparición de más signos en otras áreas de la iglesia a medida que avanzaba el proceso de desarme. Encontrar estos tallados no fue tarea fácil, puesto que muchas veces las piezas estaban cubiertas con tierra y telas de araña que rellenaban los surcos y hacían invisibles los números. Otras veces los números simplemente habían desaparecido con la pudrición. Aun así descubrimos, evidencia certera de una nomenclatura común y lógica en todas las piezas estructurales de la iglesia.

Se realizó un levantamiento planimétrico y fotográfico con todos los números encontrados y, tras el análisis de la numeración, descubrimos que los números indicaban, por un lado, el grupo estructural y su ubicación en la iglesia y, por otro lado, la posición de cada pieza dentro de este grupo.

Los grupos estructurales eran: dado (I), primera caña (II) y segunda caña (III), coro (IIII) y pórtico $(V)$ en el área de la torre. En el área de la nave, el tabique lateral izquierdo correspondía al (I), la columnata izquierda al (II), columnata derecha (III) y tabique lateral derecho (IIII). Dentro de cada uno de estos grupos, cada pieza tenía su posición determinada también por un número correlativo entre las partes, que acompañaba al número anterior. 
2. SEGUNDA CAÑA

A. Esquema planimétrico de la solera superior de la segunda caña, con sus números romanos respectivos, y su ubicación correspondiente. Todas las piezas correspondientes a la segunda caña tienen un tres (III), número que se repite en cada una de sus piezas, numeradas del I al VIII. Levantamiento realizado en obra.

B. Detalle pieza VII del cuerpo III, correspondiente a la segunda caña. Registro fotográfico en obra, archivo FAICH.

C. Solera superior segunda caña, rearmada en el suelo una vez efectuado el desarme. En esta etapa se revelaron los números romanos. Registro fotográfico en obra, archivo FAICH.

(2)

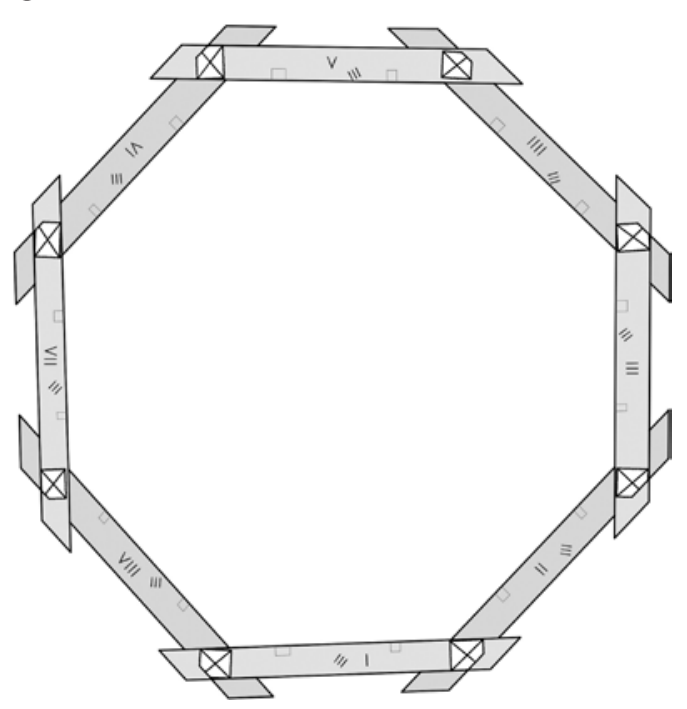

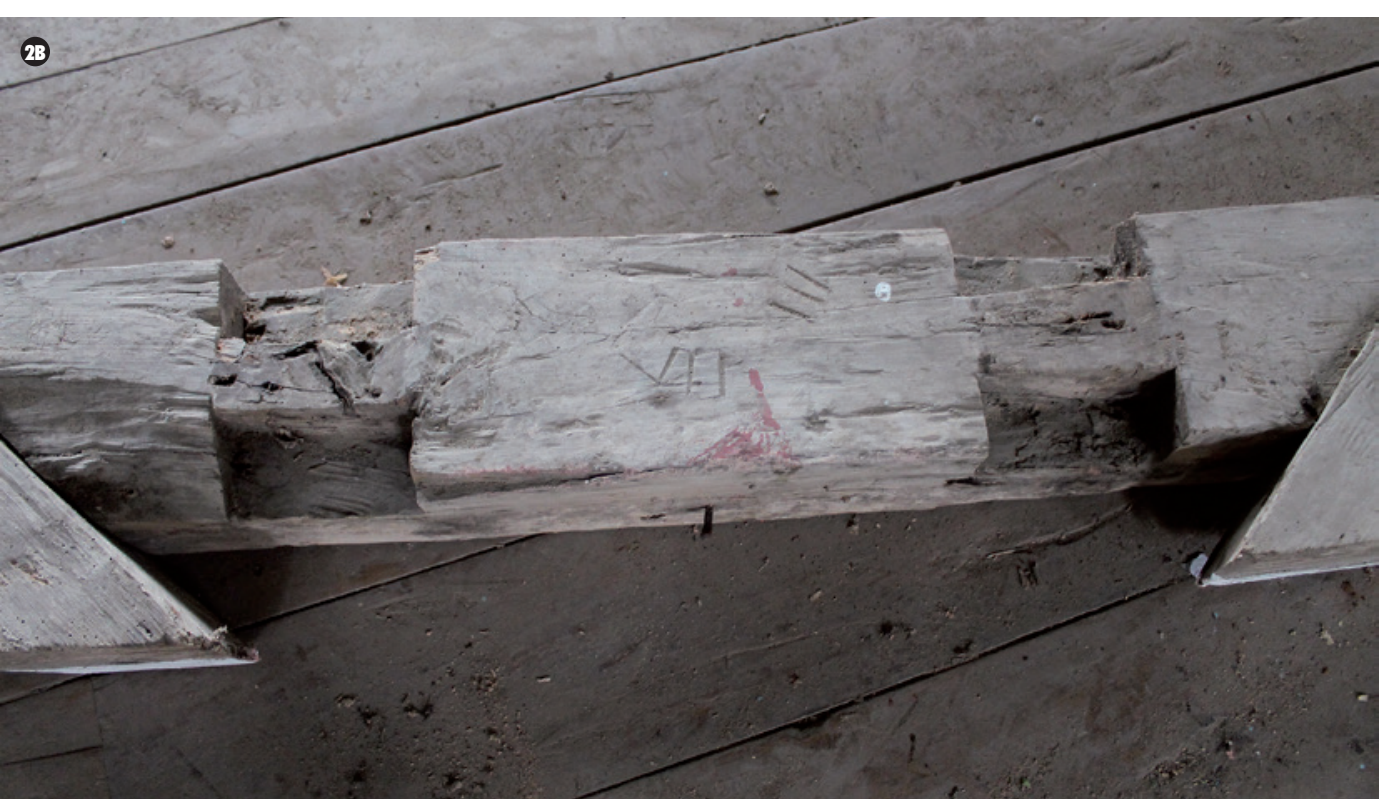

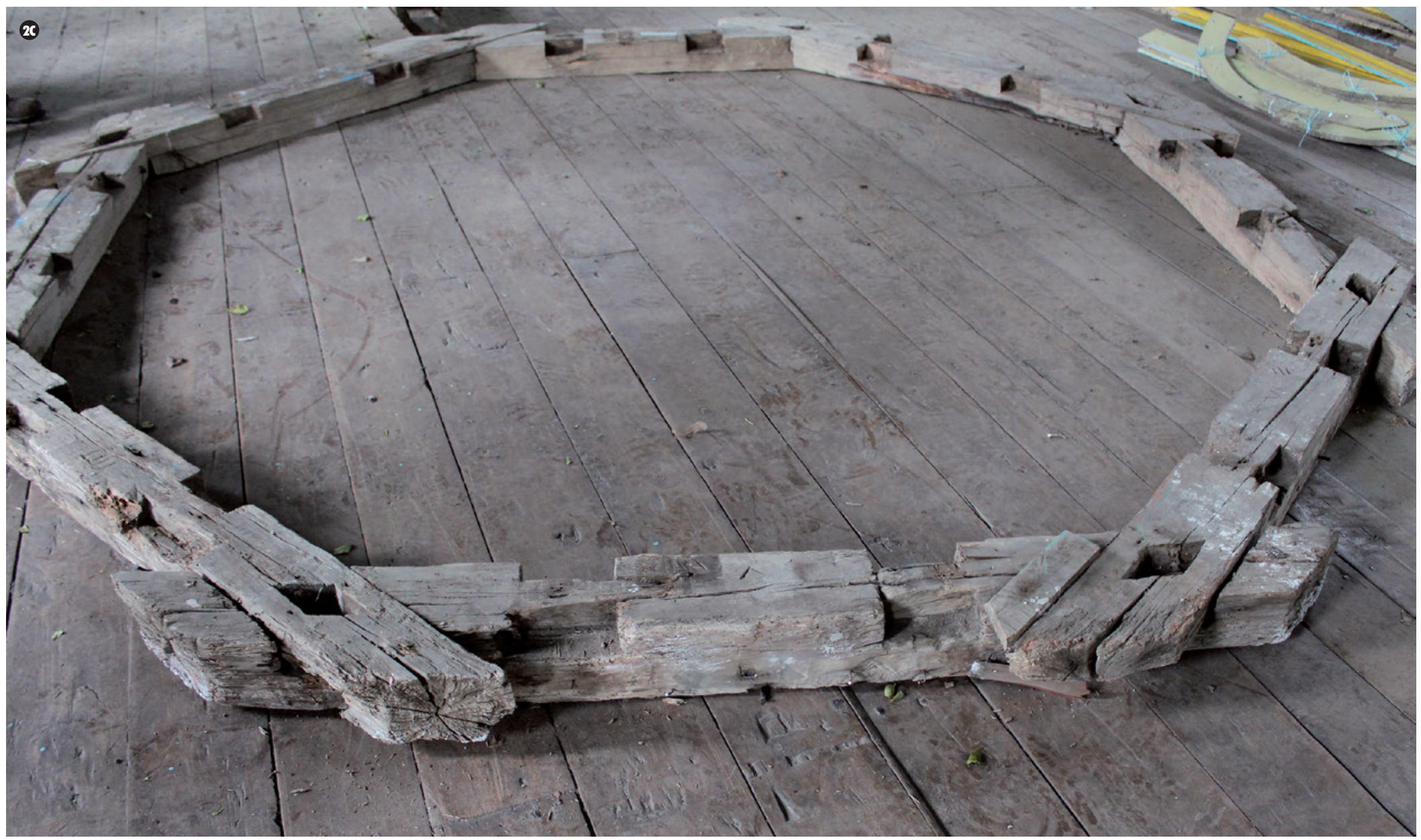


3. PRIMERA CAÑA

A. Esquema planimétrico de la solera superior de la primera caña, con sus números romanos respectivos, y su ubicación correspondiente. Todas las piezas correspondientes a la primera caña tienen un dos (II). Las piezas de la solera se numeran del I al VIII las del gato superior del I al XVI. Levantamiento planimétrico realizado en obra.

B. Detalle se la solera y envigado superior del cuerpo II, es decir, la primera caña, que a su vez muestra el estado de grave deterioro general de este cuerpo. Registro fotográfico en obra, archivo FAICH.

C. Detalle de la solera y envigado superior del cuerpo II, es decir, la primera caña. Registro fotográfico en obra, archivo FAICH

(3)

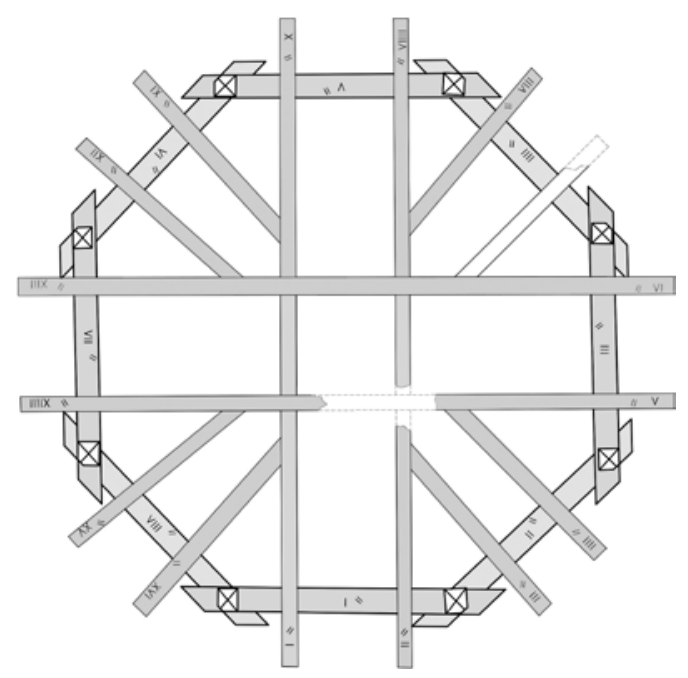

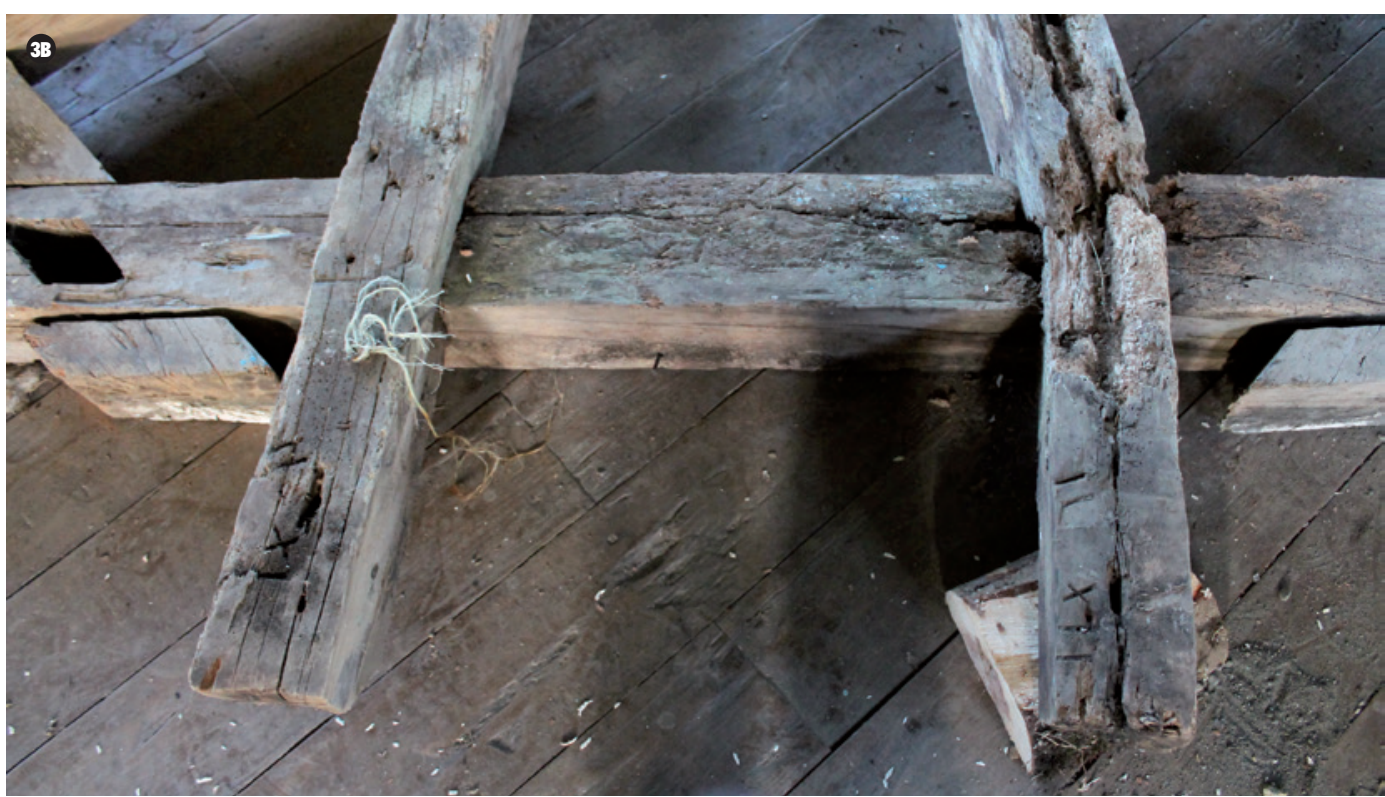

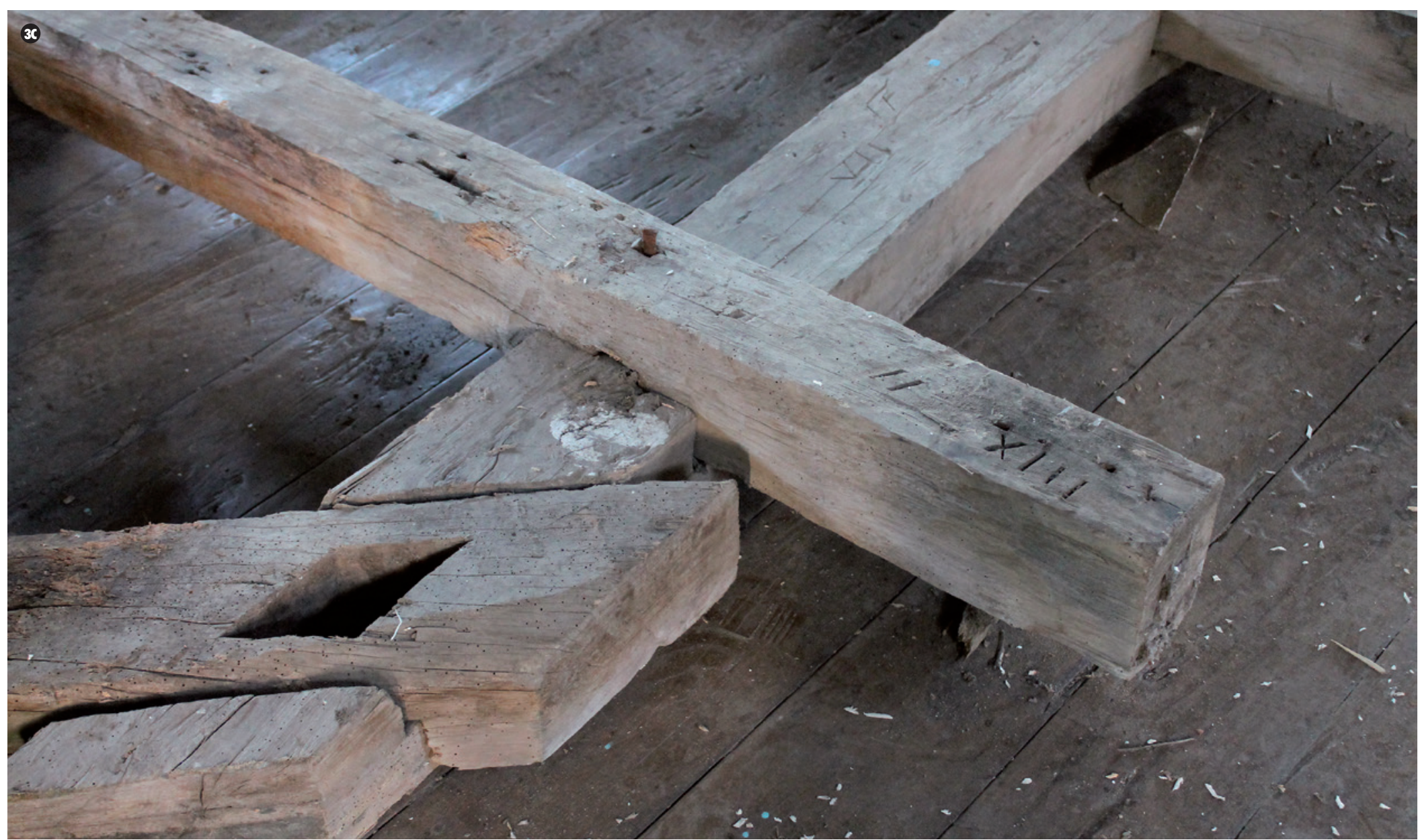


4. HASTIAL

A. Esquema planimétrico de una sección del hastial, que muestra la numeración encontrada en los pie derechos centrales del coro.

B. Se puede apreciar la numeración señalada en las distintas piezas. Registro fotográfico en obra, archivo FAICH.

C. Se puede apreciar la numeración señalada en las distintas piezas. Registro fotográfico en obra, archivo FAICH.

(1)
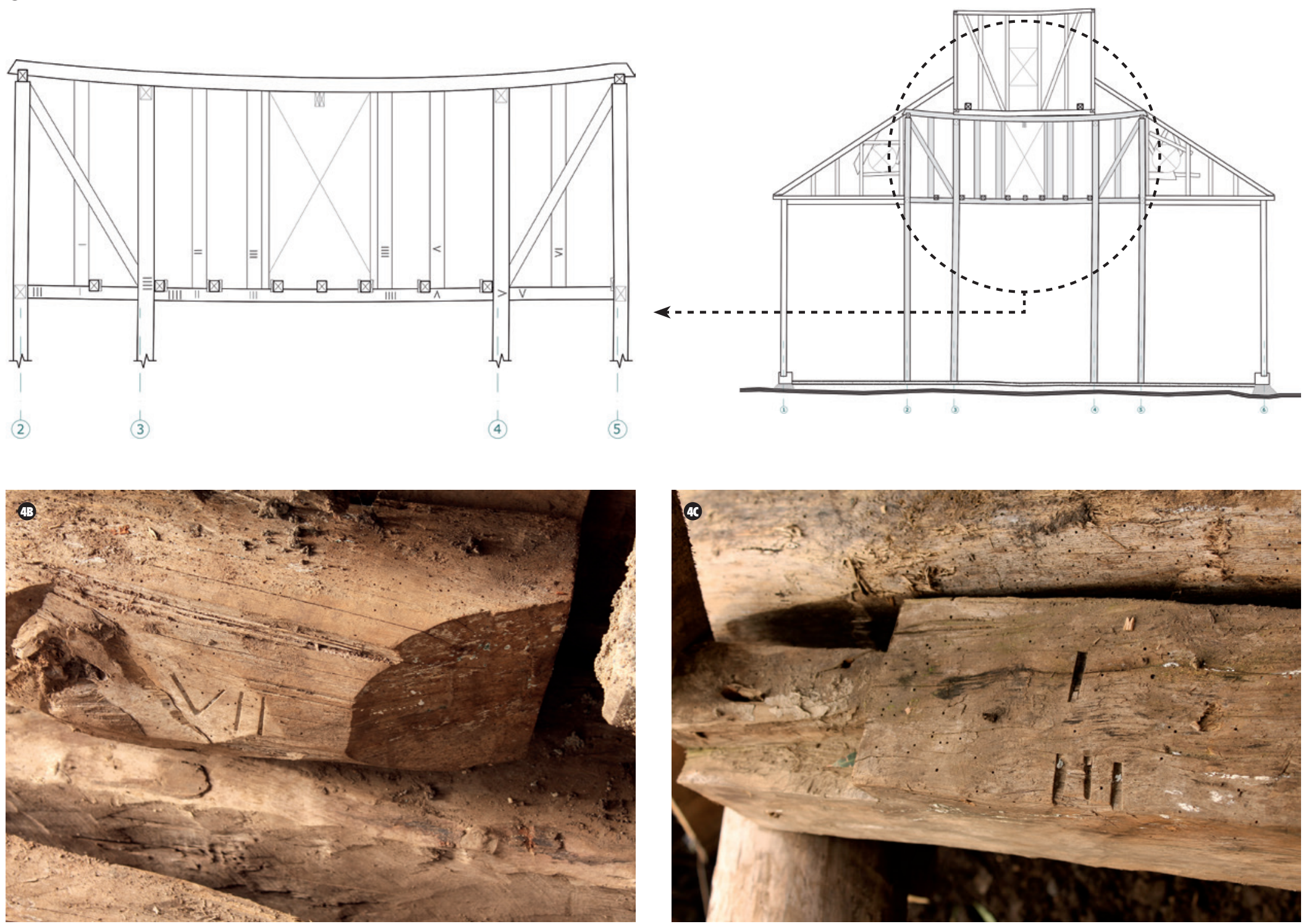

Mediante la continuación lógica de los números catastrados se pudieron reconstituir los números no encontrados. Probablemente muchas de las piezas no estaban numeradas debido a un posible reemplazo de la pieza original en ese lugar, o porque el sector donde se ubicaba dicha pieza se encontraba muy deteriorado. Este es el caso de todos los pilares que conformaban la torre-pórtico, los que fueron cortados en la sección de su base, área donde comúnmente encontramos numeración y que fueron suplidos con una pieza nueva en una intervención anterior.

Otro dato interesante es el lado hacia el cual se hallaban talladas las inscripciones en las piezas estructurales: en el caso de tabiques laterales, se encontraban hacia afuera y en el de las columnatas, se encontraban dispuestas hacia la nave central. Si bien no tenemos certeza de que este dato pueda ser relevante, puede ser un vestigio que nos ayude a dilucidar el método que utilizaban para levantar estas grandes piezas estructurales o la cronología de instalación de los grupos de estructuras.

El hecho de que cada pieza se encontrara numerada de esta manera, nos lleva a plantear la siguiente hipótesis de prefabricación:

Por un lado, creemos que este hecho podría demostrar la evolución natural de un sistema y proceso constructivo que, al basarse en 
5. Pies derechos con la inscripción de números romanos. Registro fotográfico en obra, archivo FAICH.

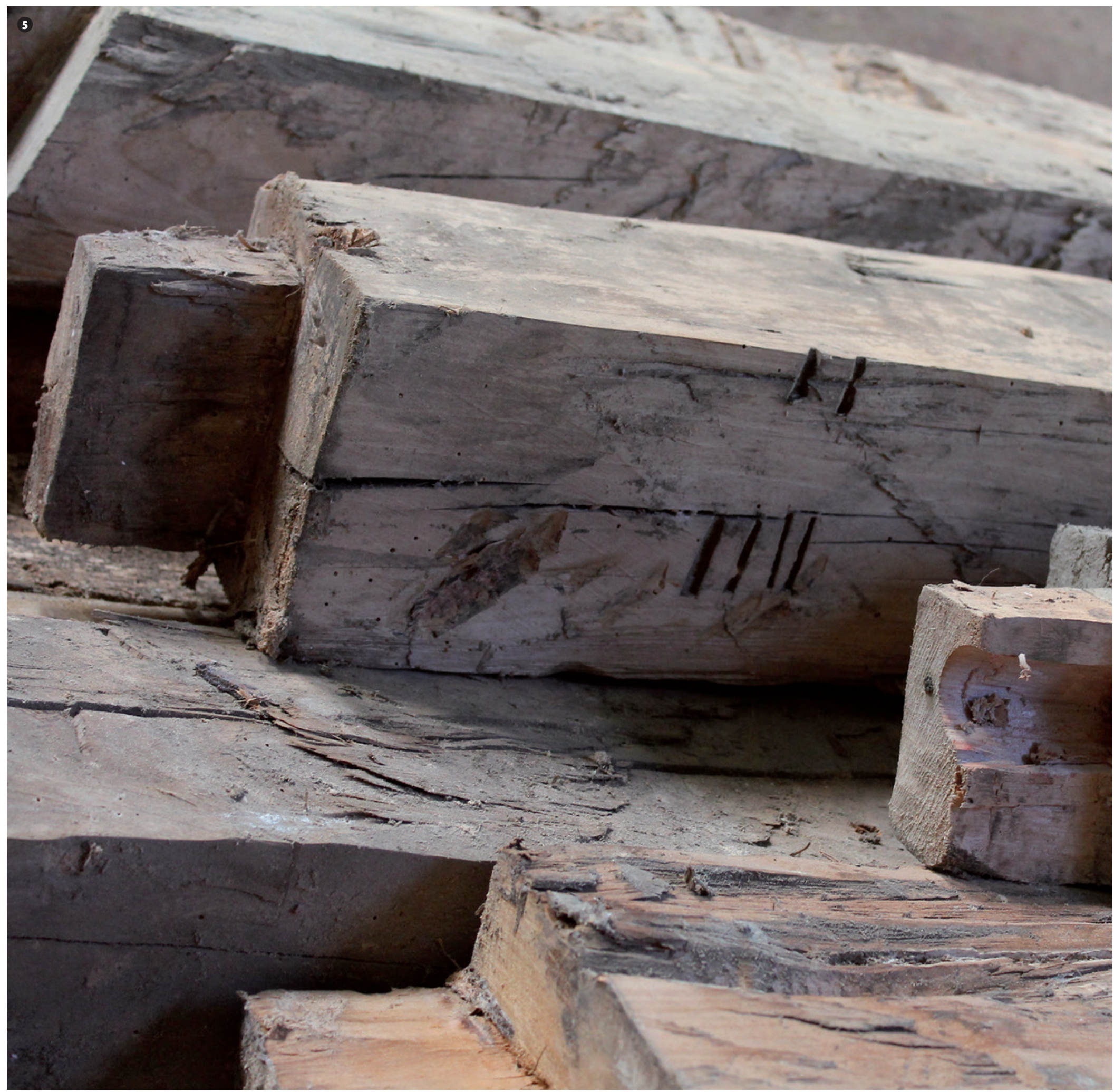


ensambles y empalmes, puede ser "armado y desarmado", lo que da la posibilidad a los carpinteros de no establecerse o permanecer durante todo el proceso de construcción en el lugar de emplazamiento definitivo de la Iglesia. Esta teoría de la prefabricación explicaría además por qué la construcción de la estructura liderada por el carpintero José Oyarzo, estuvo lista en solo 6 meses, siendo que la construcción del templo duró 12 años, según se afirma en el libro "Iglesias Misionales de Chiloé" de los autores H. Montecinos, I. Salinas y P. Basáez, investigación basada en libros parroquiales del obispado de Ancud.

El hecho de que grupos especializados dirigidos por un carpintero particular, itineraran a lo largo de la Isla construyendo iglesias a pedido, se respalda en diversos documentos históricos, especialmente libros parroquiales, en los cuales se repiten los nombres de algunos de estos carpinteros en distintas iglesias relativamente contemporáneas. Esta acción, a su vez da fuerza a la teoría de la prefabricación como un acto evolutivo lógico del un sistema constructivo altamente especializado.

Por otra parte, revisando antecedentes de situaciones similares con presencia de codificación en estructuras de otras iglesias, encontramos el caso de la iglesia de Carelmapu, cuya construcción comenzó en agosto de 1913 y ya el 2 de febrero de 1914, es decir, seis meses después, "ya estaba apta para la celebración de su fiesta religiosa más importante" (Barducci, 2002). La data de construcción es muy cercana, pero posterior a la de Rilán y también tenía tallados números romanos en su estructura, hecho que podría estar relacionado y que apoya nuestra teoría: puede ser que este mismo grupo de carpinteros sea el constructor de ambas iglesias.

Esta teoría es hoy solo una hipótesis, sin embargo, el tema merece nuestra atención y pronta investigación puesto que, de ser acertada, estamos hablando de un grado de conocimiento y evolución de la técnica superior al que creíamos. Este hallazgo nos habla de una nueva etapa de la Escuela Chilota que hasta hoy no conocemos, en que las iglesias, de ser construidas por las mismas comunidades organizadas en mingas, pasan a ser encargadas a grupos de carpinteros calificados con un alto grado de especialización, que habría llegado incluso a niveles de prefabricación en algunos templos.

\section{BIBLIOGRAFÍA}

Barducci, G. (2002). En un lugar de Chacao, de cuyo nombre no quiero acordarme. Puerto Montt: Quintessence Editora Ltda.

Montecinos, H., Basáez, P., Salinas, I. (1995). Iglesias Misionales de Chiloé. Proyecto FONDECYT 1307-90. Santiago: Departamento de Historia y Teoría de la Arquitectura, Facultad de Arquitectura y Urbanismo, Universidad de Chile. 